\title{
Mitigating Focused Ion Beam Damage in Molybdenum Nanopillars by In Situ Annealing
}

\author{
M.B. Lowry, ${ }^{* * *}$ D. Kiener, ${ }^{* * *}$ M.M. LeBlanc, ${ }^{* *}$ C. Chisholm, ${ }^{*}$ J.N. Florando, ${ }^{* *}$ J.W. Morris, \\ Jr., ${ }^{*}$ and A.M. Minor**** \\ * Department of Materials Science and Engineering, University of California, Berkeley, CA 94720 \\ ** Engineering Directorate, Lawrence Livermore National Laboratory, Livermore, CA 94550 \\ *** National Center for Electron Microscopy, Lawrence Berkeley National Laboratory, Berkeley, \\ CA 94720
}

The focused ion beam (FIB) allows for the machining of mechanical test specimens in a variety of geometries from numerous different materials. Samples range in size from nanometers to microns, and enable the study of mechanical properties in limited volumes. In particular, the last six years has seen experiments probing the effects of size on strength through flat-punch compression $[1,2]$. Further compressions performed in situ in a transmission electron microscope (TEM) have shed light on the role of microstructural defects during deformation, yielding information about hardening mechanisms such as dislocation starvation [3].

Recent work, however, has called into doubt the role of damage induced by the focused ion beam[4]. Surface defects induced by ion bombardment with $\mathrm{Ga}^{+}$, such as dislocations, Ga implantation, and amorphization, significantly alter the mechanical properties of metals. Here we show that through in situ annealing in a TEM, it is possible to at least partially remove ion bombardment damage from molybdenum nanopillars. Starting with a well-annealed, decarburized piece of single crystal molybdenum, pillars are milled by focused ion beam along a single slip [ 2920 ] orientation, as seen in Figure 1. The pillars are then annealed in situ, allowing for the observation of damage removal and any associated shape changes to the pillar. Moreover, there is a reduction in the defect density that can significantly improve the visualization of the deformation behavior during in situ compressions. Figures $2 \mathrm{a}$ and $2 \mathrm{~b}$ are bright-field and dark-field TEM images of a pillar preannealing, in which it is difficult to observe individual defect behavior. By mitigating ion bombardment damage, the utility of the focused ion beam can be recovered, thereby allowing for the production of limited volumes for quantitative mechanical testing with dramatically improved TEM visualization [5].

References

[1] Uchic, M.D., et al., Sample dimensions influence strength and crystal plasticity. Science, 2004. 305(5686): p. 986-989.

[2] Greer, J.R. and W.D. Nix, Size dependence of mechanical properties of gold at the sub-micron scale. Applied Physics a-Materials Science \& Processing, 2005. 80(8): p. 1625-1629.

[3] Shan, Z.W., et al., Mechanical annealing and source-limited deformation in submicrometrediameter Ni crystals. Nature Materials, 2008. 73(7): p. 115-119.

[4] Shim, S., et al., Effects of focused ion beam milling on the compressive behavior of directionally solidified micropillars and the nanoindentation response of an electropolished surface. Acta Materialia, 2009. 57(2): p. 503-510.

[5] This work performed under the auspices of the U.S. Department of Energy by Lawrence Livermore National Laboratory under Contract DE-AC52-07NA27344. The authors 
acknowledge support of the National Center for Electron Microscopy, Lawrence Berkeley Lab, which is supported by the U.S. Department of Energy under Contract \# DE-AC02$05 \mathrm{CH} 11231$.

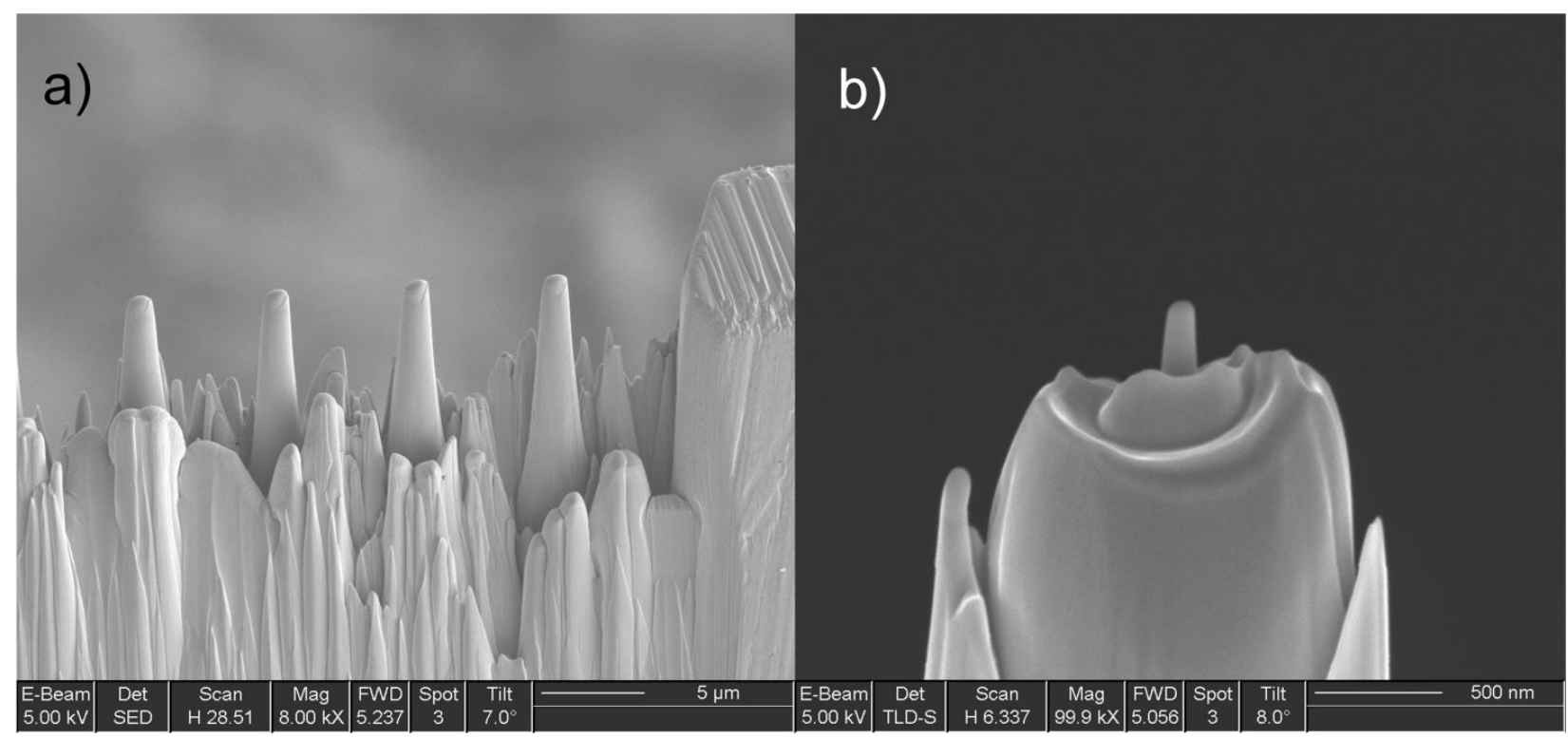

FIG. 1. (a) A row of pillars FIB machined from single crystal molybdenum. The electron transparent TEM sample is milled from the tip of the larger pillar, as seen in (b). The TEM sample is approximately $100 \mathrm{~nm}$ in diameter and $300 \mathrm{~nm}$ tall.
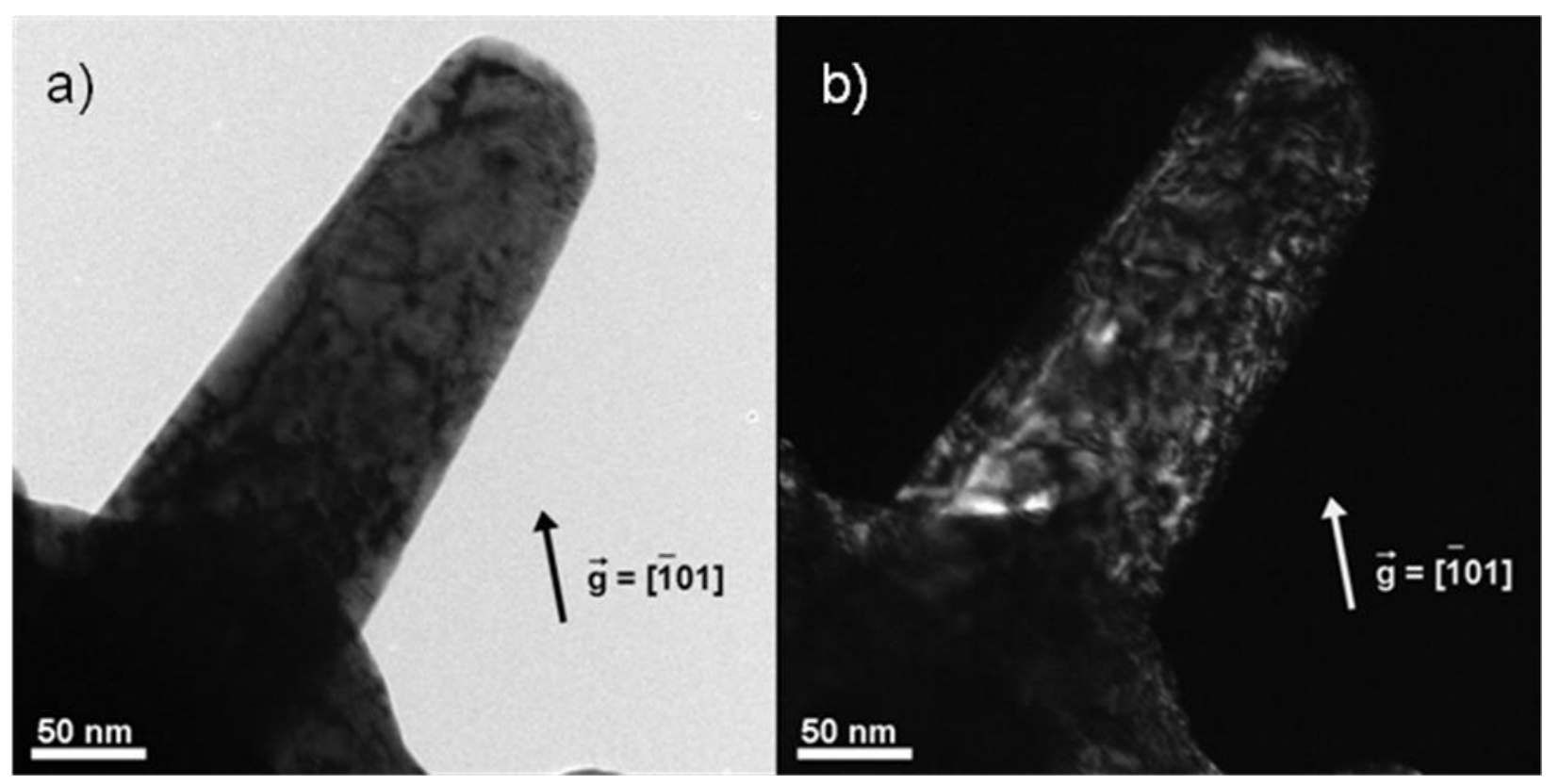

FIG. 2. (a) and (b) Bright-field and dark-field TEM images of a FIB machined single crystal molybdenum pillar, respectively. The pervasive damage from the milling process is evident in the form of dislocations, making observations during mechanical testing difficult. 